\title{
Case Report \\ Wickerhamomyces anomalous: A Rare Cause of Fungemia Causing Febrile Neutropenia in Acute Lymphoblastic Leukemia
}

\author{
Vibha Mehta, ${ }^{1}$ Aroop Mohanty ${ }^{D},{ }^{2}$ Suneeta Meena $\left(\mathbb{D},{ }^{3}\right.$ J. S. Rahul, ${ }^{4}$ Nath Uttam Kumar, ${ }^{5}$ \\ Debranjani Chattopadhyay, ${ }^{5}$ Anamika Bakliwal, ${ }^{5}$ Ranjana Choudhary, ${ }^{6}$ \\ and Pratima Gupta ${ }^{6}$
}

${ }^{1}$ PDCC Hepatic and Transplant Virology, ILBS, New Delhi, India

${ }^{2}$ Department of Clinical Microbiology, AIIMS, Gorakhpur, India

${ }^{3}$ Department of Laboratory Medicine, AIIMS, New Delhi, India

${ }^{4}$ Department of Neuroanaesthesia and Critical Care, AIIMS, New Delhi, India

${ }^{5}$ Department of Medical Oncology and Haematology, AIIMS Rishikesh, Rishikesh, India

${ }^{6}$ Department of Microbiology, AIIMS Rishikesh, Rishikesh, India

Correspondence should be addressed to Aroop Mohanty; aroopmohanty7785@yahoo.com and Suneeta Meena; suneetameena@ gmail.com

Received 23 September 2020; Revised 13 December 2020; Accepted 21 December 2020; Published 29 December 2020

Academic Editor: Mohd Adnan

Copyright (C) 2020 Vibha Mehta et al. This is an open access article distributed under the Creative Commons Attribution License, which permits unrestricted use, distribution, and reproduction in any medium, provided the original work is properly cited.

Candida bloodstream infection is the major cause of increased morbidity and mortality (20-49\%) in hospitalized patients in both paediatric and adult age groups. Due to the increase in the number of immunocompromised patients, other important species such as Trichosporon asahii and Debaryomyces hansenii are emerging. One such organism, Wickerhamomyces anomalous, previously known as Pichia anomala (teleomorph stages of several Candida species), is increasingly being reported as a cause of fungemia in neonatal intensive care units and is now increasingly being reported in a lot of immunosuppressive conditions such as interstitial lung disease, endocarditis, enteritis, corticosteroids, and chemotherapy uptake. Though this yeast is ubiquitous in nature, systemic infections from isolated cases and sporadic outbreaks with high mortality have been reported in ICUs, which emphasize the importance to consider this fungus within the diagnostic possibilities. Here, we report a case of catheter-related bloodstream infection (CRBSI) caused by W. anomalus in a leukemic immunosuppressed patient who was successfully treated by early detection and treatment of this emerging fungus.

\section{Introduction}

Candida bloodstream infection is the primary cause of increased morbidity and mortality (20-49\%) in hospitalized patients in both paediatric and adult age groups worldwide. Despite advancements in the early diagnosis and treatment, Candida species rank fourth in the United States and seventh in Europe in causing bloodstream infections (BSIs) [1]. In India, few studies have reported varying candidemia rates from 4 to as high as $18 \%$ with a constant increase in isolation of non-albicans Candida (NAC) from BSIs [1]. Initially, only Candida albicans were considered to be the most crucial yeast species to cause invasive disseminated infection; however, due to an increase in the number of immunocompromised patients, other important yeast species such as Trichosporon asahii and Debaryomyces hansenii are emerging rampantly [2].

Wickerhamomyces anomalus (W. anomalus), previously known as Hansenula anomala (H. anomala), was later on included as a synonym in the genus Pichia and was referred to as Pichia anomala [3, 4]. It is the teleomorph stage of several Candida species and is found mainly in plants, fruits, and oil. The first report about $H$. anomala isolation from the lungs of an infant was published in 1958 by Wang and Schwarz [5]. In India, it was first responsible for causing an outbreak in the neonatal intensive care unit of a tertiary care 
centre in 1996. It is now being increasingly reported in a lot of immunosuppressive conditions such as interstitial lung disease, endocarditis, enteritis, corticosteroids, and chemotherapy uptake [6]. Though this yeast is ubiquitous in nature, systemic infections from isolated cases and sporadic outbreaks with high mortality have been reported in ICUs, which emphasize the importance to consider this fungus within the diagnostic possibilities [6]. Here, we report a case of catheter-related bloodstream infection (CRBSI) caused by $W$. anomalus in a leukemic immunosuppressed patient who was successfully treated by early detection and treatment of this emerging fungus.

\section{Case Report}

A 36-year-old male, follow-up case of B-cell acute lymphoblastic leukemia on cyclophosphamide and methotrexate immunosuppressive therapy, presented to the emergency department of our tertiary care centre with the chief complaint of fever with chills and rigors for two days. The fever started four days after the last chemotherapy session. The patient had an implanted long-term central venous catheter. Previous chemotherapy sessions were uneventful. Radiographic and laboratory evaluations (including CBC, LFT, and RFT) were unremarkable. Blood culture from both central catheter and peripheral line was collected and sent to the microbiology laboratory. He was treated empirically with injective piperacillin-tazobactam 3.375 gm IV 6 hourly and injection vancomycin 1 gm IV 12 hourly. After 22 hours of incubation, the BACTEC blood culture system (Becton Dickinson and Company, USA) signalled positive in both central catheter and peripheral line. However, the central line came out to be positive one hour before the peripheral line. Direct Gram stain from both positive blood culture bottles revealed budding yeast cells (Figure 1). The specimen was subcultured onto blood agar, chocolate agar, MacConkey agar, and Sabouraud's dextrose agar (SDA) at $27^{\circ} \mathrm{C}$ and $34^{\circ} \mathrm{C}$, respectively. Within 24 hours, white to cream-colored, glabrous yeast-like colonies of $W$. anomalus were formed on SDA at $37^{\circ} \mathrm{C}$ (Figure 2). Conventional methods such as lactophenol cotton blue (LPCB) mount and cornmeal agar (CMA) showed spherical to ellipsoidal budding yeast cells with abundant pseudohyphae. The germ tube test came out to be negative. CHROMagar Candida showed the presence of pink mucoid colonies of $W$. anomalus grown after 48 hours at $37^{\circ} \mathrm{C}$ (Figure 3). It was further identified by MALDI-TOF MS (Bruker Daltonics, Bremen, Germany) as Wickerhamomyces anomalus with a confidence interval (CI) of 2.19. Hence, a clinical diagnosis of $W$. anomalus catheter-related bloodstream infection (CRBSI) was established. Fluconazole ( $800 \mathrm{mg}$ on the first day followed by $400 \mathrm{mg}$ O.D.) was started, and antibiotics were de-escalated. The central venous catheter and peripheral line were removed immediately, and the tip culture was sent for fungal culture, which also yielded positive growth for W. anomalus. No other focus of Wickerhamomyces could be noticed. After three days of antifungal therapy, the patient clinically improved and became afebrile. A follow-

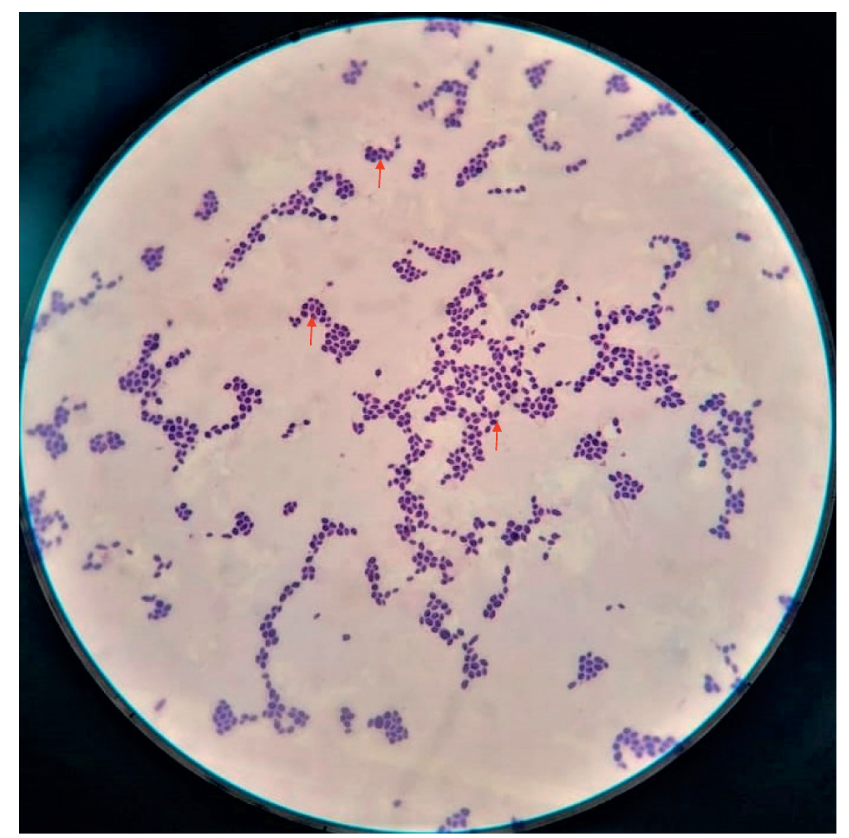

FIGURE 1: Direct Gram stain from both positive blood culture bottles revealed budding yeast cells (BYCs).

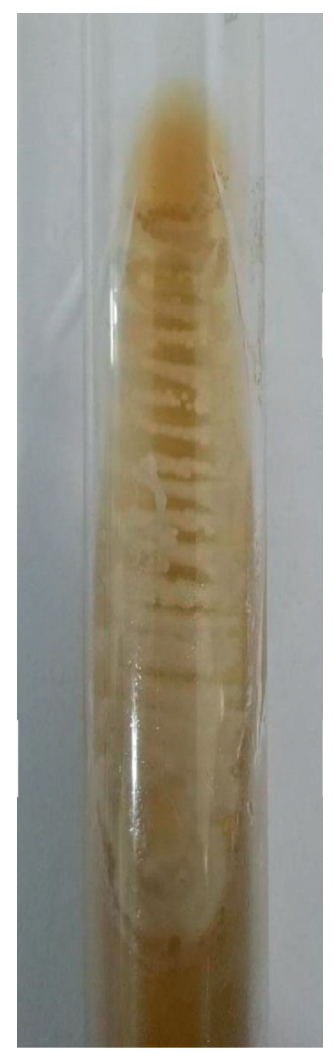

FIgURE 2: White to cream-colored, glabrous yeast-like colonies of $W$. anomalus were formed on SDA at $37^{\circ} \mathrm{C}$ after 24 hours of incubation.

up blood culture after two weeks was performed, which came out to be sterile, and as a result, the patient was discharged under satisfactory conditions. 


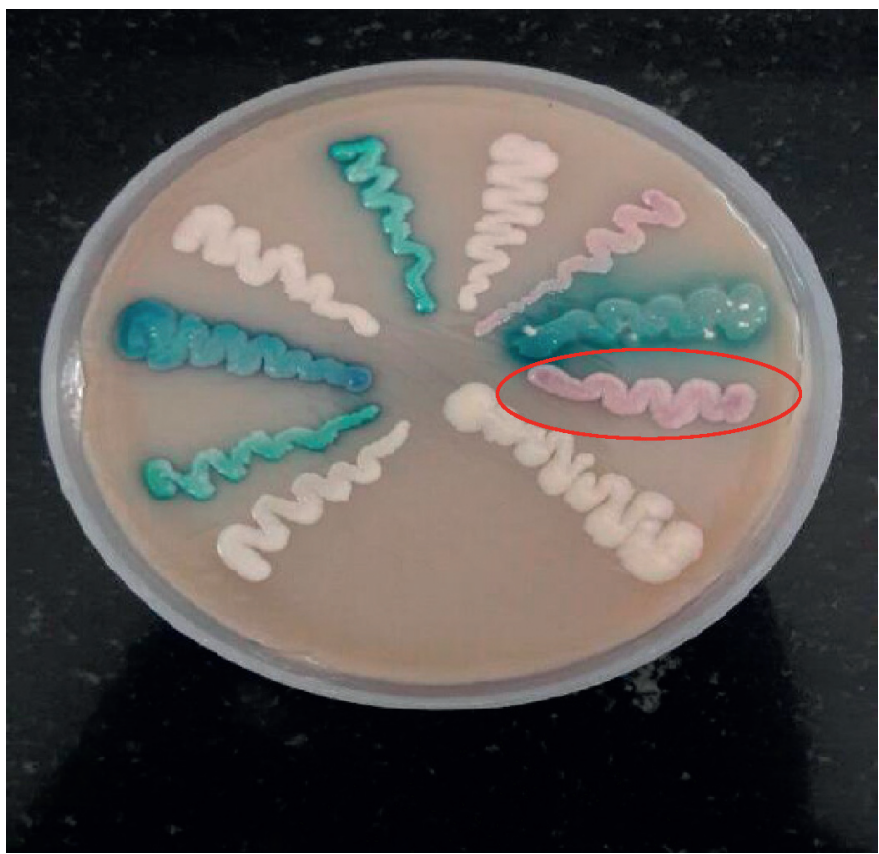

Figure 3: CHROM agar Candida showed the presence of pink mucoid colonies of $W$. anomalus grown after 48 hours at $37^{\circ} \mathrm{C}$.

\section{Discussion}

Candida albicans has always been in the limelight as essential yeast species to cause human invasive infections. However, due to increased immunosuppressive conditions and advanced methods for detection and identification of yeasts, many other clinically critical opportunistic species are reported with varying antifungal susceptibility [7].

$W$. anomalus is a ubiquitous organism and was initially thought to be a plant pathogen, but recently, it has been increasingly reported as a causative agent of fungemia in both immunocompetent and immunocompromised patients $[8,9]$. It has been reported as a causative agent of nosocomial fungemia in neonatal intensive care units, critical care units, and AIDS patients. It has also been implicated in nosocomial cerebral ventriculitis in low-birthweight neonates, endocarditis in intravenous drug abusers, and urinary tract infection in renal transplant recipients. Cross-contamination, through the hands of caregivers, has also been incriminated as an important cause in the past [10-13]. In our case, the presence of an implanted central venous catheter in the adult patient on chemotherapy could be a possible contributory risk factor for the fungus to grow.

The literature on the susceptibility profile of $W$. anomalus is limited; though based on the susceptibility profiles of most isolates, it appears to be very much similar to that of Candida glabrata [14]. Several studies were carried out to analyse the antifungal susceptibility of clinical isolates belonging to this uncommon species of Candida. One study showed that out of fifteen $W$. anomalus strains, eight were resistant to fluconazole, six to itraconazole, and ketoconazole to both, and one was flucytosine resistant [15].

Da Matta et al. were able to publish the most extensive susceptibility profile of such isolates from patients presenting with nosocomial fungemia where they reported MICs of 16, 1, 0.5, 0.25, and 1 to fluconazole, itraconazole, voriconazole, caspofungin, and amphotericin $\mathrm{B}$, respectively. A series of case reports by Chitasombat et al. describes one isolate of $W$. anomalus that was susceptible to the azoles, amphotericin B, and echinocandins [16].

These findings are quite worrisome since besides amphotericin B, only a few therapeutic options are currently effective against this rare fungal infection. Although antifungal susceptibility testing could not be performed in our case, our patient was treated successfully with fluconazole therapy for 14 days.

This case further puts a much-needed light on $W$. anomalus and its ability to cause invasive infection with varying antifungal susceptibility. It is rather challenging to identify $W$. anomalus by conventional as well as widely used commercial yeast diagnostic kits such as API 20C AUX, ID32C, and Vitek-2 and commonly misidentified as Candida utilis by commercial systems [17].

In our patient, the pathogen was identified as W. anomalus by MALDI-TOF MS (Bruker Daltonics, Bremen, Germany) which yielded the results in a few minutes and helped the clinicians in early initiation of the therapy of choice and improving the prognosis in this case. Molecular identification was not possible in this case due to limited resources and facilities. Our experience with these rare yeasts is that antifungal susceptibility-driven therapy is the key to management of such pathogens.

Our case report demonstrates the importance of early suspicion, organism identification, and differentiation of ascomycetous yeast species, specifically in immunosuppressed high-risk patient populations such as those with total parenteral nutrition, term and preterm NICU patients, chronic indwelling intravenous access, and compromised 
immune systems. An effective antifungal prophylaxis policy may be necessary for these patients when one or more risk factors exist.

\section{Conclusion}

With an emerging number of fungal pathogens in these patient populations that are not reliably sensitive to azoles, it is increasingly necessary to cultivate a healthy suspicion for echinocandins and azole-resistant organisms that may further require susceptibility testing. So, early suspicion, detection, and treatment can be very helpful in curbing these emerging pathogens.

\section{Conflicts of Interest}

The authors declare that they have no conflicts of interest.

\section{References}

[1] P. Bhattacharjee, "Epidemiology and antifungal susceptibility of Candida species in a tertiary care hospital, Kolkata, India," Current Medical Mycology, vol. 2, no. 2, pp. 20-27, 2016.

[2] C. R. Paula, V. L. Krebs, M. E. Auler et al., "Nosocomial infection in newborns by Pichia anomala in a Brazilian intensive care unit," Medical Mycology, vol. 44, no. 5, pp. 479-484, 2006.

[3] C. P. Kurtzman, "Synonymy of the yeast genera Hansenula and Pichia demonstrated through comparisons of deoxyribonucleic acid relatedness," Antonie Van Leeuwenhoek, vol. 50, no. 3, pp. 209-217, 1984.

[4] C. P. Kurtzman, "DNA relatedness among phenotypically similar species of Pichia," Mycologia, vol. 84, no. 1, pp. 72-76, 1992.

[5] C. J. K. Wang and J. Schwarz, "The etiology of interstitial pneumonia," Mycopathologia et Mycologia Applicata, vol. 9, pp. 299-306, 1958.

[6] A. Chakrabarti, K. Singh, A. Narang et al., "Outbreak of Pichia anomala infection in the pediatric service of a tertiary care center in northern India," Journal of Clinical Microbiology, vol. 39, pp. 1702-1706, 2001.

[7] K. C. Hazen, "New and emerging yeast pathogens," Clinical Microbiology Reviews, vol. 8, no. 4, pp. 462-478, 1995.

[8] E. Haron, E. Anaissie, F. Dumphy, K. Mc credie, and V. Fainstein, "Hansenula anomala fungemia," Reviews of Infectious Diseases, vol. 10, no. 6, pp. 1182-1186, 1988.

[9] A. S. Klein, G. T. Tortora, R. Malowitz, and W. H. Greene, "Hansenula anomala: a new fungal pathogen. Two case reports and a review of the literature," Archives of Internal Medicine, vol. 148, no. 5, pp. 1210-1213, 1988.

[10] A. Kalkanci, M. Dizbay, O. Turan et al., "Nosocomial transmission of Candida pelliculosa fungemia in a pediatric intensive care unit and review of the literature," The Turkish Journal of Pediatrics, vol. 52, pp. 42-49, 2010.

[11] R. Salesa, A. Burgos, C. Fernandez-Mazarrasa, G. Quindos, and J. Ponton, "Transient fungaemia due to Candida pelliculosa in a patient with AIDS," Mycoses, vol. 34, no. 7-8, pp. 327-329, 1991.

[12] N. Murphy, C. R. Buchanan, V. Damjanovic, R. Whitaker, C. A. Hart, and R. W. Cooke, "Infection and colonization of neonates by Hansenula anomala," Lancet, vol. 1, no. 8476, pp. 291-293, 1986.
[13] B. Nohinek, C. S. Zee-Cheng, W. G. Barnes, L. Dall, and H. R. Gibbs, "Infective endocarditis of a bicuspid aortic valve caused by Hansenula anomala," The American Journal of Medicine, vol. 82, no. 1, pp. 165-168, 1987.

[14] V. L. Da Matta, M. S. C. De Melhem, A. L. Colombo et al., "Antifungal drug susceptibility profile of Pichia anomala isolated from patients presenting with nosocomial fungemia," Antimicrobial Agents and Chemotherapy, vol. 51, no. 4, pp. 1573-1576, 2007.

[15] F. Barchiesi, A. M. Tortorano, L. F. Di Francesco, M. Cogliati, G. Scalise, and M. A. Viviani, "In-vitro activity of five antifungal agents against uncommon clinical isolates of Candida spp," Journal of Antimicrobial Chemotherapy, vol. 43, no. 2, pp. 295-299, 1999.

[16] M. N. Chitasombat, D. P. Kofteridis, Y. Jiang, J. Tarrand, R. E. Lewis, and D. P. Kontoyiannis, "Rare opportunistic (non-Candida, non-Cryptococcus) yeast bloodstream infections in patients with cancer," Journal of Infection, vol. 64, no. 1, pp. 68-75, 2012.

[17] A. W. Chan, E. J. Cartwright, S. C. Redd, C. S. Kraft, and Y. F. Wang, "Pichia anomala (Candida pelliculosa) Fungemia in a patient with sickle cell disease," Mycopathologia, vol. 176, no. 3-4, pp. 273-277, 2013. 